\title{
Correction to: Effects of Maternal Exercise During Pregnancy on Perinatal Growth and Childhood Obesity Outcomes: A Meta-analysis and Meta-regression
}

\author{
Yanting Chen ${ }^{1,3} \cdot$ Guiling $\mathrm{Ma}^{2} \cdot$ Yun $\mathrm{Hu}^{2} \cdot$ Qiyuan Yang ${ }^{4}$ Jeanene M. Deavila ${ }^{1,3} \cdot$ Mei-Jun Zhu ${ }^{5} \cdot$ Min Du $^{1,3}$ (1)
}

Published online: 9 July 2021

(c) Springer Nature Switzerland AG 2021

\section{Correction to: Sports Medicine}

$$
\text { https://doi.org/10.1007/s40279-021-01499-6 }
$$

Reference numbering errors occurred in the text and

Figs. 3-5 during the typesetting process of this article.

The original article has been updated.

The original article can be found online at https://doi.org/10.1007/ s40279-021-01499-6.

Min Du

min.du@wsu.edu

1 Center for Reproductive Biology, School of Molecular Biosciences, Washington State University, Pullman,

WA 99164, USA

2 College of Animal Science and Technology, Yangzhou University, Yangzhou 225009, People's Republic of China

3 Nutrigenoimics and Growth Biology Laboratory, Department of Animal Sciences, Washington State University, Pullman, WA 99164, USA

4 Department of Molecular, Cell and Cancer Biology, University of Massachusetts Medical School, Worcester, MA 01655, USA

5 School of Food Science, Washington State University, Pullman, WA 99164, USA 